\title{
Prognostic Impact of Calcified Plaque Morphology After Drug Eluting Stent Implantation - An Optical Coherence Tomography Study -
}

\author{
Saki Iwai, MD; Makoto Watanabe, MD, PhD; Akihiko Okamura, MD; Atsushi Kyodo, MD; \\ Kazutaka Nogi, MD; Daisuke Kamon, MD; Yukihiro Hashimoto, MD; Tomoya Ueda, MD, PhD; \\ Tsunenari Soeda, MD, PhD; Hiroyuki Okura, MD, PhD; Yoshihiko Saito, MD, PhD
}

\begin{abstract}
Background: Optical coherence tomography (OCT) has the potential to characterize the detailed morphology of calcified coronary plaques. This study examined the prognostic impact of calcified plaque morphology in patients with coronary artery calcification (CAC) who underwent newer-generation drug-eluting stent (DES) implantation.

Methods and Results: In all, 251 patients with moderate to severe CAC who underwent OCT-guided DES implantation were reviewed retrospectively and divided into 3 groups according to OCT findings of the target lesion: 25 patients (10.0\%) with calcified nodules (CN), 69 patients (27.5\%) with calcified protrusion (CP) without CN, and 157 patients (62.5\%) with superficial calcific sheet (SC) without $\mathrm{CN}$ and CP. The primary endpoint was major adverse cardiac events (MACE), defined as a composite of cardiac death, myocardial infarction, and target lesion revascularization (TLR). Kaplan-Meier survival analysis revealed that, among the 3 groups, the rates of MACE-free survival (log-rank test, $P=0.0117$ ), myocardial infarction (log-rank test, $P=0.0103$ ), and TLR (log-rank test, $\mathrm{P}=0.0455$ ) were significantly worse in patients with $\mathrm{CN}$. Multivariate Cox proportional hazards analysis demonstrated that $\mathrm{CN}$ was an independent predictor of MACE (hazard ratio 4.41; 95\% confidence interval 1.63-10.8; $P=0.0047$ ).
\end{abstract}

Conclusions: Target lesion CN was associated with higher cardiac event rates in patients who underwent newer-generation DES implantation for lesions with moderate to severe CAC.

Key Words: Calcified coronary plaques; Calcified nodules; Optical coherence tomography

D rug-eluting stents (DES) achieve superior clinical and angiographic outcomes in patients with coronary artery disease undergoing percutaneous coronary intervention (PCI) than bare-metal stents (BMS). ${ }^{1}$ However, coronary artery calcification (CAC) may limit stent delivery and expansion ${ }^{2}$ and damage the polymer or drug coating, resulting in impaired drug delivery to the vessel wall and restenosis or stent thrombosis, despite the use of a scoring balloon or rotational atherectomy. ${ }^{36} \mathrm{~A}$ previous study demonstrated that moderate to severe CAC on angiography was strongly associated with stent thrombosis and target lesion revascularization (TLR) after DES implantation. ${ }^{7}$ However, intravascular imaging studies have not clarified how calcified plaque morphology affects the clinical outcomes following DES implantation.

Optical coherence tomography (OCT) is an intravascular imaging modality with a maximal spatial resolution of $10 \mathrm{~mm}$, which is 10 -fold higher than that of intravascular

\section{Editorial $\mathrm{p} ? ? ? ?$}

ultrasound (IVUS). ${ }^{\mathbf{8} 9}$ Because near-infrared light from the OCT system can penetrate calcium, OCT provides a more detailed characterization of the morphology of calcified coronary plaques than IVUS.,4,10 A recent OCT study classified calcified plaques responsible for acute coronary syndrome (ACS) into 3 groups: calcified nodule $(\mathrm{CN})$, calcified protrusion $(\mathrm{CP})$, and superficial calcific sheet (SC). ${ }^{11} \mathrm{CN}$ and $\mathrm{CP}$ present a similar protruding calcific mass into the lumen and convex luminal shape, unlike SC. $\mathrm{CN}$ often shows an irregular leading edge due to a cluster of small calcific nodules, whereas CP typically shows a smooth leading edge. ${ }^{11}$ Although it has been reported that these different morphologies of calcified plaque may result in different post-stent OCT findings, ${ }^{12}$ the effects of the 3 calcified plaque subtypes on long-term clinical outcomes

Received December 7, 2020; revised manuscript received March 17, 2021; accepted March 31, 2021; J-STAGE Advance Publication released online May 27, 2021 Time for primary review: 21 days

Department of Cardiovascular Medicine, Nara Medical University, Kashihara (S.I., M.W., A.O., A.K., K.N., D.K., Y.H., T.U., T.S., Y.S.); Department of Cardiology, Gifu University Graduate School of Medicine, Gifu (H.O.), Japan

Mailing address: Makoto Watanabe, MD, PhD, Department of Cardiovascular Medicine, Nara Medical University, 840 Shijo-cho, Kashihara634-8522, Japan. E-mail: wmakoto07@kcn.jp

All rights are reserved to the Japanese Circulation Society. For permissions, please e-mail: cj@j-circ.or.jp

ISSN-1346-9843 


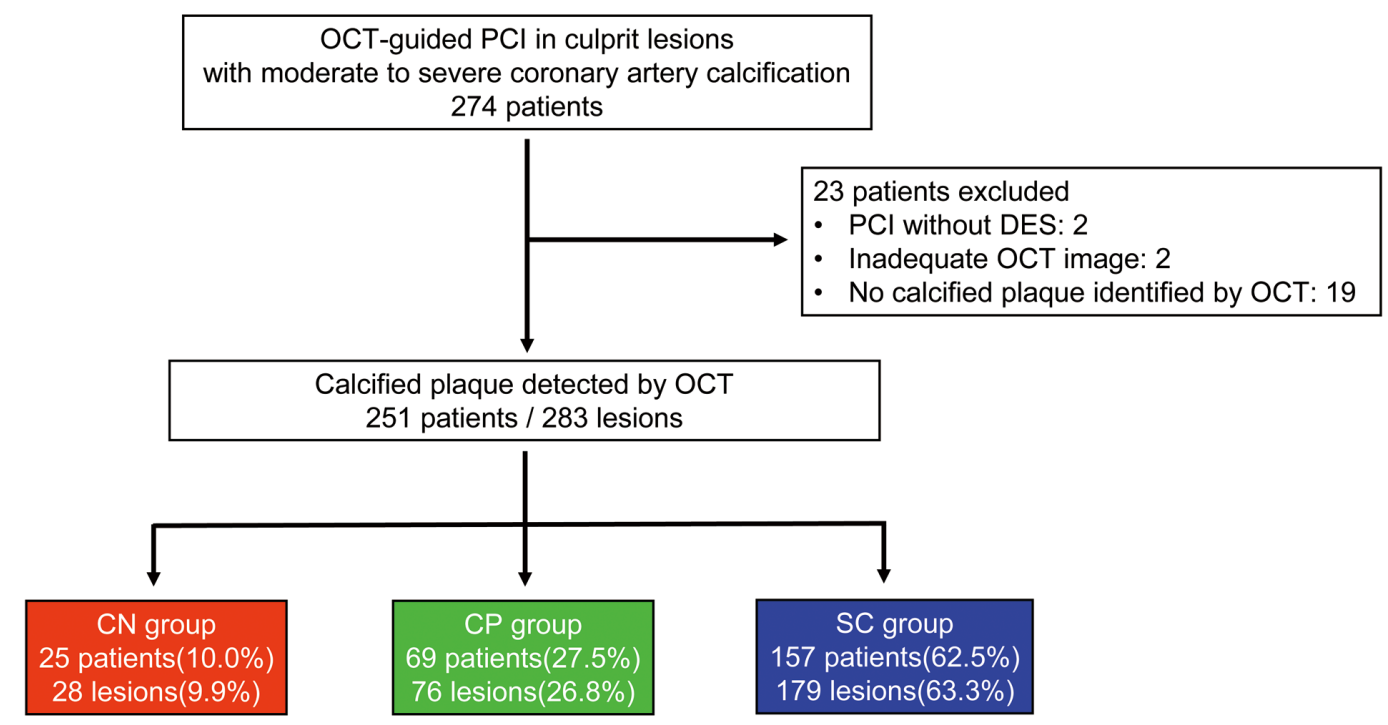

Figure 1. Study flowchart. CN, calcified nodule; CP, calcified protrusion; DES, drug-eluting stent; OCT, optical coherence tomography; $\mathrm{PCl}$, percutaneous coronary intervention; $\mathrm{SC}$, superficial calcified sheet.

after DES implantation remain unclear.

The aim of this study was to investigate the prognostic impact of calcified plaque morphology on cardiovascular events in patients with moderate to severe CAC who underwent OCT-guided DES implantation.

\section{Methods}

\section{Study Population}

This was a single-center retrospective observational study conducted at Nara Medical University, Japan. In all, 274 patients with moderate to severe CAC in de novo target lesions on fluoroscopy who underwent OCT-guided PCI between January 2014 and December 2016 were enrolled in the study. In this study, we retrospectively analyzed patients who fulfilled the following criteria: (1) implantation with a second- or third-generation DES; (2) OCT performed before stenting; (3) OCT images analyzable; and (4) calcified plaques detected by OCT. Twenty-three lesions were excluded because DES were not used $(n=2)$, baseline OCT images were inadequate $(n=2)$, or there was an absence of target lesion calcification on OCT $(n=19)$. This left 251 patients (283 lesions) with target lesion calcification on OCT who were included and analyzed in this study (Figure 1).

The diagnosis of ACS included ST-elevation myocardial infarction (STEMI), non-ST-elevation myocardial infarction (NSTEMI), and unstable angina pectoris (UAP). STEMI was defined as continuous chest pain, ST-segment elevation in 2 contiguous leads or new left bundle-branch block on 12-lead electrocardiography, and elevated cardiac marker levels (creatine kinase-MB or troponin). NSTEMI was defined as ischemic symptoms in the absence of STsegment elevation on the electrocardiogram with elevated cardiac marker levels. UAP was defined as the presence of newly developed or accelerating chest symptoms on exertion or rest angina within 2 weeks of presentation without biomarker release. ${ }^{13}$
This study was approved by the Ethics Committee of Nara Medical University (Reference no. 1759-2) and complied with the Declaration of Helsinki Ethical Principles for Medical Research Involving Human Subjects. Informed consent was obtained in the form of an opt-out option on the Department of Cardiovascular Medicine, Nara Medical University website.

\section{Diagnostic Coronary Angiography and Procedures}

Diagnostic coronary angiography was performed in all patients after intracoronary administration of isosorbide dinitrate $(1 \mathrm{mg})$. The severity of calcification was classified based on the radiopaque densities prior to contrast injection as either moderate (radiopaque densities noted only during the cardiac cycle and typically involving only 1 side of the vascular wall) or severe (radiopaque density noted without cardiac motion and generally involving both sides of the arterial wall). ${ }^{7}$ PCI was performed using standard techniques and catheters via the femoral or radial approach according to the operator's or center's usual practice. All lesions were treated with second- or third-generation DES. After the index PCI, patients were maintained on aspirin (100 mg once daily) and ticlopidine (200 mg twice daily) or clopidogrel ( $75 \mathrm{mg}$ once daily) for at least 1 year unless they had risk factors for bleeding.

\section{OCT Image Acquisition}

OCT imaging was performed using a frequency domain OCT system (C8 System, Dragonfly imaging catheter and ILUMIEN OPTIS; St. Jude Medical, St. Paul, MN, USA) or an optical frequency domain imaging (OFDI) system (Terumo, Tokyo, Japan). Briefly, the OCT imaging catheter was advanced distal to the target lesion over a conventional 0.014-inch angioplasty guidewire through a 6- or 7-Fr guiding catheter. After intracoronary administration of $1 \mathrm{mg}$ isosorbide dinitrate, pullbacks were performed during continuous injection of X-ray contrast medium or low-molecular-weight dextran containing lactate Ringer's 

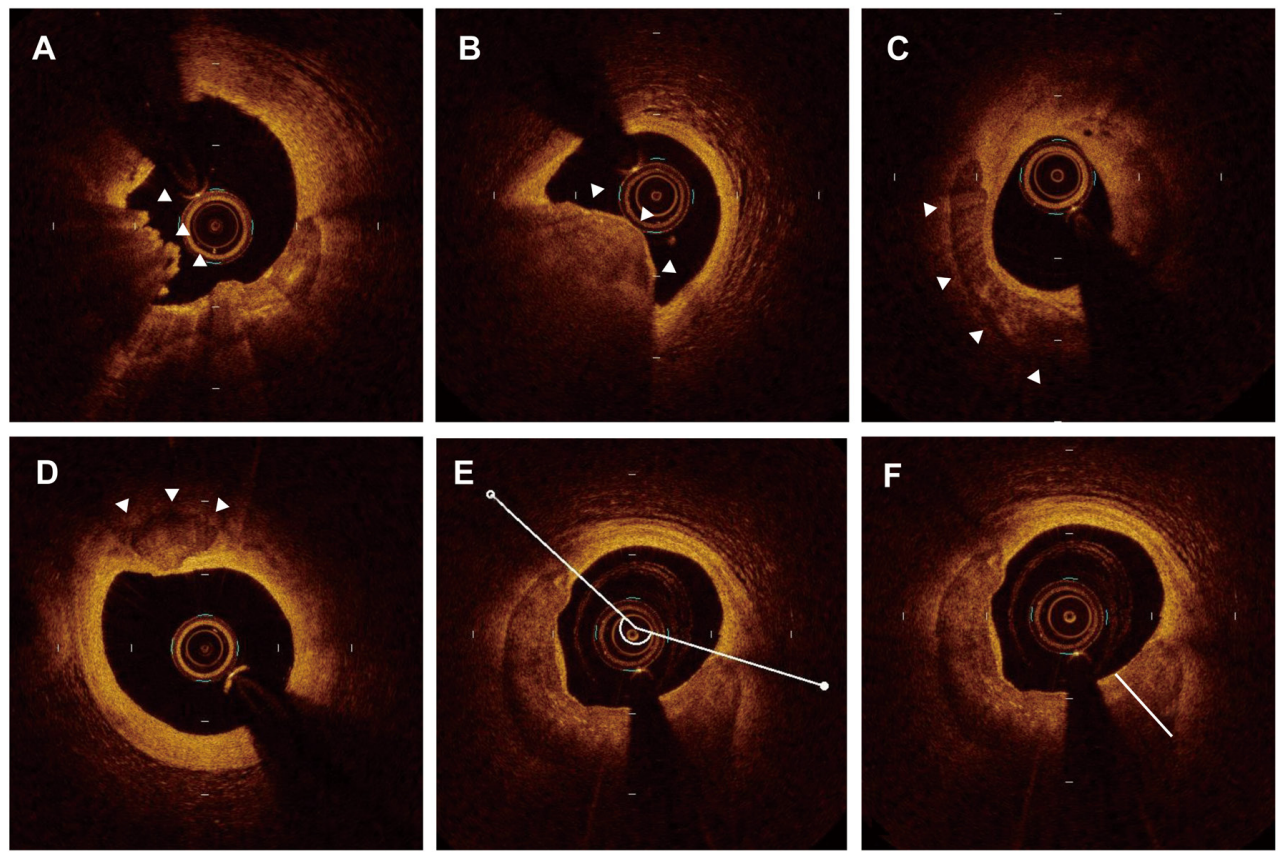

Figure 2. Representative optical coherence tomography (OCT) images of calcified plaques. (A) A calcified nodule (CN) appears as an expulsion of a cluster of small calcified nodules into the lumen (arrowheads). (B) A calcified protrusion (CP) appears as a protruding calcific mass without small eruptive calcific nodules (arrowheads). (C) A superficial calcific sheet (SC) appears as a sheet-like superficial calcific plate with minimal or no disruption of the overlying fibrous tissue and without erupted nodules or masses protruding into the lumen (arrowheads). (D) Spotty calcification appears as spotty calcium deposits (arrowheads) with length $<4 \mathrm{~mm}$ and maximal arc $<90^{\circ}$. (E) Calculation of the calcium arc; the calcium arc (white line) was 205.2 $2^{\circ}$. (F) Calculation of calcium thickness; the calcium thickness (white line) was $0.78 \mathrm{~mm}$.

solution at a rate of $2.5-3.5 \mathrm{~mL} / \mathrm{s}$ through the guiding catheter using an injection pump to remove blood from the field of view and enable clear visualization of the vessel wall. Images were acquired at a rate of 5 frames $/ \mathrm{mm}$ and an automated pullback speed of $20 \mathrm{~mm} / \mathrm{s}^{14}$

\section{OCT Image Analysis}

OCT images were analyzed by 2 independent observers (S.I. and M.W.) in a blinded manner using a dedicated offline review system (St. Jude Medical). Two observers blinded to each patient's clinical and lesion characteristics independently performed the OCT measurements. Any discordance between the 2 observers was resolved by consensus with a third reviewer (A.O.).

A baseline OCT examination was performed before the PCI to evaluate the morphology of the calcified plaque within the target lesion. In the case of lesions with severe narrowing precluding passage of the OCT or OFDI catheter or removal of blood from the field for clear visualization of the vessel wall, or lesions with an angiographically visible thrombus, the baseline OCT examination was performed after predilatation using a balloon catheter, rotational atherectomy, or thrombectomy.

The target lesion was defined as the segment that was stented by comparing baseline and post-PCI OCT images. A calcified plaque was defined as a low-signal intensity area with sharply delineated borders. ${ }^{15}$ Calcified plaques were classified into 3 types: $\mathrm{CN}, \mathrm{CP}$, and $\mathrm{SC} .{ }^{11} \mathrm{CN}$ was defined as a high-backscattering mass protruding into the lumen with strong signal attenuation and an irregular surface (Figure 2A). CP, also called protruding nodular calcification, was defined as a protruding calcific mass without eruptive nodules (Figure 2B). SC was defined as a non-protruding calcified plaque without $\mathrm{CN}$ or $\mathrm{CP}($ Figure $2 \mathrm{C}, \mathrm{D})$. $^{11,16}$ If the target lesion contained $\mathrm{CN}$ along with other types of calcified plaque, the calcified plaque was assigned to the $\mathrm{CN}$ group. If the target lesion contained $\mathrm{CP}$ along with $\mathrm{SC}$, the calcified plaque was assigned to the $\mathrm{CP}$ group. The culprit lesions in the first implanted stent were analyzed as target lesions in patients with multivessel PCI.

Each calcific plaque in the target lesion was quantitatively evaluated according to the following parameters: maximum calcium arc, maximum calcium thickness, and calcium length. ${ }^{17}$ The maximum calcium arc was determined as the largest calcium arc within the target lesion by visual screening (Figure 2E). Maximum calcium thickness was measured at the thickest calcium segment on the frame with the thickest calcium within the target lesion by visual screening (Figure 2F). When the calcific deposit was thick and the border was not clear due to attenuation, the maximum visible thickness was measured. Calcium length was calculated based on the number of frames with calcified plaques. When $\geq 2$ calcified plaques were present in the target lesion, the plaque with the maximum calcium arc was selected for quantitative analysis.

Minimum lumen area (MLA), minimum stent area (MSA), stent length, mean reference lumen area, stent edge dissection (SED), incomplete stent apposition (ISA), and 


\begin{tabular}{|c|c|c|c|c|}
\hline & $\begin{array}{c}C N \\
(n=25)\end{array}$ & $\begin{array}{c}C P \\
(n=69)\end{array}$ & $\begin{array}{c}S C \\
(n=157)\end{array}$ & $P$ value \\
\hline Age & $70.6 \pm 10.7$ & $71.9 \pm 10.4$ & $72.0 \pm 9.4$ & 0.8092 \\
\hline Male sex & $19(76.0)$ & $53(76.8)$ & $119(75.8)$ & 0.9864 \\
\hline Smoker & $16(64.0)$ & $46(67.7)$ & $116(74.8)$ & 0.3613 \\
\hline Diabetes & $11(44.0)$ & $34(49.3)$ & $68(43.3)$ & 0.7046 \\
\hline Dyslipidemia & $14(56.0)$ & $51(73.9)$ & $114(72.6)$ & 0.1996 \\
\hline Hypertension & $22(88.0)$ & $60(87.0)$ & $129(82.2)$ & 0.5648 \\
\hline Maintenance dialysis & $11(44.0)^{*, \dagger}$ & $9(13.0)$ & $13(8.3)$ & $<0.0001$ \\
\hline History of MI & $1(4.0)$ & $15(21.7)$ & $23(14.7)$ & 0.0977 \\
\hline History of stroke & $3(12.0)$ & $13(18.8)$ & $16(10.2)$ & 0.1981 \\
\hline Peripheral artery disease & $5(20.0)$ & $9(13.0)$ & $16(10.2)$ & 0.3536 \\
\hline \multicolumn{5}{|l|}{ Laboratory data } \\
\hline Serum creatinine $(\mathrm{mg} / \mathrm{dL})$ & $1.19[0.70-6.62]$ & $0.92[0.77-1.09]$ & $0.91[0.74-1.13]$ & 0.1334 \\
\hline eGFR $\left(\mathrm{mL} / \mathrm{min} / 1.73 \mathrm{~m}^{2}\right)$ & $36.8[7.2-72.1]$ & $59.2[47.6-71.1]$ & $60.5[46.1-75.5]^{*}$ & 0.0555 \\
\hline $\mathrm{HDL}-\mathrm{C}(\mathrm{mg} / \mathrm{dL})$ & $49.5[38-59]$ & $49[40-58]$ & $48[40-58]$ & 0.9325 \\
\hline LDL-C (mg/dL) & $84[70-116]$ & $91[78-111]$ & 101 [77-127] & 0.2768 \\
\hline $\mathrm{HbA1c}(\%)$ & $5.7[5.3-6.7]$ & $6.2[5.7-7.0]$ & $6.1[5.7-6.8]$ & 0.3063 \\
\hline Serum corrected $\mathrm{Ca}(\mathrm{mg} / \mathrm{dL})$ & $8.9[8.6-9.0]$ & $8.9[8.6-9.2]$ & $8.8[8.6-9.1]$ & 0.2296 \\
\hline Serum $P(m g / d L)$ & $3.5[3.0-5.6]$ & 3.5 [3.0-3.9] & $3.2[2.9-3.6]$ & 0.0465 \\
\hline \multicolumn{5}{|l|}{ Medications on admission } \\
\hline Insulin & $2(8.0)$ & $11(15.9)$ & $13(8.3)$ & 0.2022 \\
\hline Statin & $11(44.0)$ & $36(52.9)$ & $72(46.2)$ & 0.5962 \\
\hline Vitamin $\mathrm{K}$ antagonist & $2(8.0)$ & $3(4.4)$ & $7(4.5)$ & 0.7358 \\
\hline \multicolumn{5}{|l|}{ Medications at discharge } \\
\hline Aspirin & $25(100)$ & $69(100)$ & $154(99.4)$ & 0.7375 \\
\hline Thienopyridine & $25(100)$ & $69(100)$ & $153(98.7)$ & 0.5426 \\
\hline ACEI/ARB & $16(64.0)$ & $58(84.1)$ & $119(76.8)$ & 0.1129 \\
\hline$\beta$-blocker & $14(56.0)$ & $35(50.7)$ & $91(58.7)$ & 0.5386 \\
\hline Statin & $15(60.0)^{*, \dagger}$ & $58(84.1)$ & $128(82.6)$ & 0.0209 \\
\hline Insulin & $1(4.0)$ & $11(15.9)$ & $17(11.0)$ & 0.2557 \\
\hline Oral hypoglycemia agent & $7(28.0)$ & $26(37.7)$ & $49(31.6)$ & 0.5764 \\
\hline
\end{tabular}

Unless indicated otherwise, values are presented as the mean $\pm S D$, median [interquartile range], or $n(\%) .{ }^{*}<0.0167$ compared with the calcified protrusion (CP) group; ${ }^{\dagger} P<0.0167$ compared with the superficial calcific sheet (SC) group. ACEI, angiotensin-converting enzyme inhibitor; ARB, angiotensin receptor blocker; CN, calcified nodule; eGFR, estimated glomerular filtration rate; HDL-C, high-density lipoprotein cholesterol; LDL-C, low-density lipoprotein cholesterol; Ml, myocardial infarction; $\mathrm{P}$, phosphorus.

stent underexpansion were evaluated using post-PCI OCT imaging after the final procedure. ${ }^{18}$ SED was defined as the disruption of the vessel luminal surface by a visible flap at the stent edge or in reference segments $5 \mathrm{~mm}$ proximal and distal to the stent. ISA was defined as separation of the inner surface of a stent strut from the inner vessel wall, in segments without a side branch, by a distance greater than or equal to the axial resolution of OCT plus the width of the stent strut of each stent type, including the polymer coating. When stent struts with ISA were detected, we measured the ISA distance, defined as the distance between the inner surface of the stent strut and the inner surface of the vessel wall. The maximum ISA distance was determined as the longest distance in 3 candidate frames selected by visual screening of all cross-sectional images of a stented segment. Post-PCI mean reference lumen area was defined as the mean of the largest lumen area within $5 \mathrm{~mm}$ of the proximal and distal stent edges. The stent expansion ratio was defined as the ratio of MSA to the postprocedural mean reference area. ${ }^{18}$ Stent underexpansion was defined as a stent expansion ratio $<0.8 .{ }^{18}$

\section{Endpoints}

The primary endpoint of the study was major adverse cardiovascular events (MACE), which included cardiac death, myocardial infarction (spontaneous or periprocedural), and TLR. The secondary endpoints were all-cause death, cardiac death, myocardial infarction, TLR, and target vessel revascularization (TVR). Myocardial infarction included periprocedural infarctions, which were defined as an absolute increase in creatinine kinase myocardial band (CK$\mathrm{MB}) \geq 10$ times the upper reference limit. ${ }^{19}$ The indication of TLR/TVR or other PCI was considered for the culprit lesions for ACS or the target lesions $\geq 50 \%$ in diameter stenosis by qualitative coronary angiographic assessment taking into consideration the results of preprocedural myocardial perfusion scintigraphy, exercise electrocardiography, or fractional flow reserve.

All patients were followed-up at Nara Medical University or other primary care institutions every 1-3 months after discharge. Angiographic follow-up was scheduled at 8-12 months after PCI but was performed earlier if there was clinical indication of ischemia. Clinical outcomes were 


\begin{tabular}{|c|c|c|c|c|}
\hline & $\begin{array}{c}C N \\
(n=28)\end{array}$ & $\begin{array}{c}C P \\
(n=76)\end{array}$ & $\underset{(n=179)}{S C}$ & $P$ value \\
\hline Clinical presentation at $\mathrm{PCl}$ & & & & 0.2550 \\
\hline STEMI & $6(21.4)$ & $13(17.1)$ & $45(25.1)$ & \\
\hline NSTEMI & $1(3.6)$ & $3(4.0)$ & $17(9.5)$ & \\
\hline Unstable angina pectoris & $3(10.7)$ & $5(6.6)$ & $18(10.1)$ & \\
\hline Stable coronary artery disease & $18(64.3)$ & $55(72.4)$ & 99 (55.3) & \\
\hline Culprit lesion for ACS & $10(35.7)$ & $21(27.6)^{\dagger}$ & $80(44.7)$ & 0.0355 \\
\hline Location of target lesions & & & & 0.0008 \\
\hline Right coronary artery & $14(50.0)^{\star, \dagger}$ & $16(21.1)$ & $37(20.7)$ & \\
\hline Left anterior descending artery & $10(35.7)$ & $55(72.4)$ & $123(68.7)$ & \\
\hline Left circumflex artery & $1(3.6)$ & $3(4.0)$ & $16(8.9)$ & \\
\hline Left main trunk & $3(10.7)$ & $2(2.6)$ & $3(1.7)$ & \\
\hline Severe calcification & $21(75.0)^{\dagger}$ & $38(50.0)^{\dagger}$ & $41(22.9)$ & $<0.0001$ \\
\hline Use of rotational atherectomy & $16(57.1)^{\star, \dagger}$ & $13(17.1)$ & $13(7.3)$ & $<0.0001$ \\
\hline Stent diameter $(\mathrm{mm})$ & $3.25[2.75-3.5]^{\star, \dagger}$ & $2.625[2.5-3.0]$ & $2.75[2.5-3.0]$ & 0.0008 \\
\hline Stent length $(\mathrm{mm})$ & $33[18-48]$ & $33[27-51]^{\dagger}$ & 28 [18-42] & 0.0119 \\
\hline
\end{tabular}

Unless indicated otherwise, values are presented as the median [interquartile range] or $n(\%)$. ${ }^{*}<0.0167$ compared with the calcified protrusion (CP) group; ${ }^{\mathrm{P}}<0.0167$ compared with the superficial calcific sheet (SC) group. ACS, acute coronary syndrome; CN, calcified nodule; NSTEMI, non-ST-elevation myocardial infarction; PCI, percutaneous coronary intervention; STEMI, ST-elevation myocardial infarction.

\begin{tabular}{|c|c|c|c|c|}
\hline & $\begin{array}{c}C N \\
(n=28)\end{array}$ & $\underset{(n=76)}{C P}$ & $\underset{(n=179)}{S C}$ & $P$ value \\
\hline \multicolumn{5}{|l|}{ Before PCI } \\
\hline Maximum calcium arc $\left(^{\circ}\right)$ & $229.5[177.4-324.4]^{\dagger}$ & $221.2[137.9-329.3]^{\dagger}$ & $161.8[110.2-261.5]$ & 0.0007 \\
\hline Maximum calcium thickness $(\mathrm{mm})$ & $1.17 \pm 0.30^{\dagger}$ & $1.10 \pm 0.24^{\dagger}$ & $0.98 \pm 0.28$ & 0.0001 \\
\hline Calcium length $(\mathrm{mm})$ & $18.3[11.6-31.2]^{*, \dagger}$ & $11.6[6.6-18.4]^{\dagger}$ & $6.9[4.6-11.0]$ & $<0.0001$ \\
\hline \multicolumn{5}{|l|}{ After PCI } \\
\hline Minimum lumen area $\left(\mathrm{mm}^{2}\right)$ & $5.45[3.61-6.77]$ & $4.50[3.31-5.39]$ & $4.41[3.54-5.70]$ & 0.1423 \\
\hline Minimum stent area $\left(\mathrm{mm}^{2}\right)$ & $5.45[3.6-6.70]$ & $4.3[3.31-5.35]$ & $4.39[3.53-5.70]$ & 0.0732 \\
\hline Reference lumen area $\left(\mathrm{mm}^{2}\right)$ & $8.17[6.14-10.3]^{*, \dagger}$ & $5.77[4.81-7.16]$ & $6.21[5.03-7.92]$ & 0.0014 \\
\hline Stent expansion ratio & $0.68[0.57-0.79]$ & $0.74[0.64-0.84]$ & $0.74[0.64-0.82]$ & 0.1158 \\
\hline Stent under-expansion & $20(76.9)$ & $43(59.7)$ & $121(68.8)$ & 0.2090 \\
\hline Total stent length (mm) & $34.4[26.1-45.5]$ & $33.0[24.5-44.8]^{\dagger}$ & $28.0[19.2-38.0]$ & 0.0055 \\
\hline Stent edge dissection & $12(50 \%)^{\dagger}$ & $24(33.8)$ & 39 (22.9) & 0.0109 \\
\hline Proximal edge dissection & $8(33.3)^{\dagger}$ & $14(19.7)$ & $21(12.3)$ & 0.0204 \\
\hline Distal edge dissection & $4(16.0)$ & $11(15.9)$ & $22(12.9)$ & 0.7957 \\
\hline ISA & $24(96.0)$ & 67 (91.8) & $138(79.3)$ & 0.0117 \\
\hline Maximum ISA distance $(\mu \mathrm{m})$ & $530[340-715]^{\dagger}$ & $360[270-560]$ & $340[270-490]$ & 0.0203 \\
\hline
\end{tabular}

Unless indicated otherwise, values are presented as the mean $\pm \mathrm{SD}$, medians [interquartile range], or $\mathrm{n}(\%)$. ${ }^{\mathrm{P}} \mathrm{P}<0.0167$ compared with the $\mathrm{CP}$ group; ${ }^{\dagger} \mathrm{P}<0.0167$ compared with the SC group. ISA, incomplete stent apposition. Other abbreviations as in Table 2.

assessed from clinical records at Nara Medical University. All patients were enrolled in the study regardless of the follow-up duration and were censored at the time of death or last known consultation at Nara Medical University.

\section{Statistical Analysis}

All analyses were performed using JMP version 11 (SAS Institute, Cary, NC, USA). Continuous variables are expressed as the mean $\pm \mathrm{SD}$ or median with interquartile range (IQR), whereas categorical variables are presented as counts and percentages. Categorical data were compared using the Pearson $X^{2}$ test. Continuous variables were compared using parametric 1-way analysis of variance or the non-parametric Kruskal-Wallis test based on the variable's distribution. The cumulative incidence of survivalfree periods from clinical events was estimated using the Kaplan-Meier method. In the case of significant differences, pairwise post hoc tests were performed with Bonferroni correction. A univariate Cox proportional hazards model was used to compare primary and secondary endpoints between patients with and without $\mathrm{CN}$. A multivariate Cox proportional hazards model was used to identify independent predictors of the primary endpoint, which included variables with $\mathrm{P}<0.05$ in the univariate model. A $\mathrm{P}<0.05$ was considered statistically significant. 

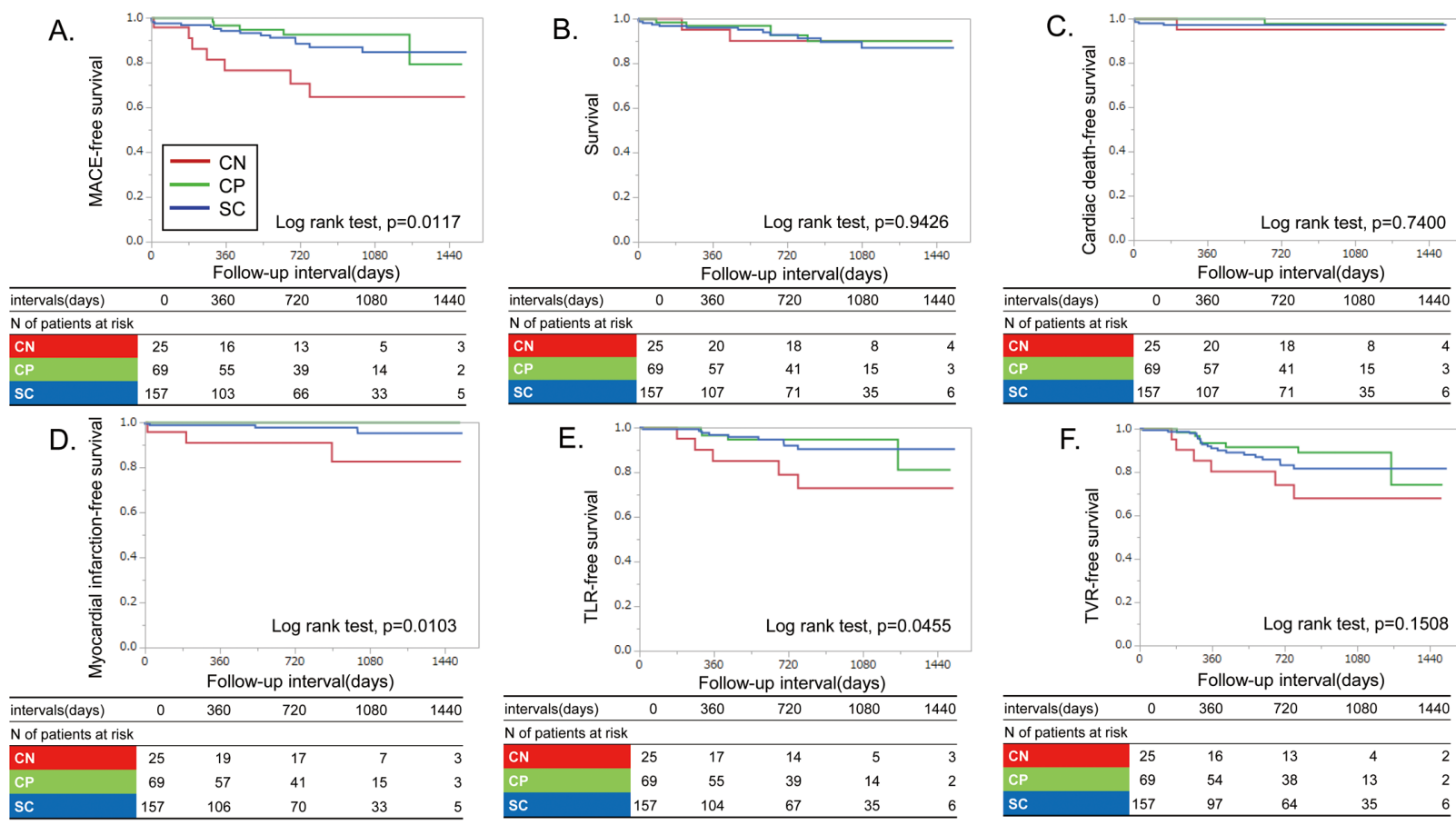

Figure 3. Kaplan-Meier survival curves of clinical outcomes. (A) Primary endpoint-free survival; (B) all cause death-free survival; (C) cardiac death-free survival; (D) myocardial infarction-free survival, (E) target lesion revascularization (TLR)-free survival, and (F) target vessel revascularization (TVR)-free survival. CN, calcified nodule; CP, calcified protrusion; SC, superficial calcific sheet.

\section{Results}

In all, 251 patients who met the inclusion criteria were analyzed in this study. Twenty-five patients $(10.0 \%)$ were classified into the $\mathrm{CN}$ group, $69(27.5 \%)$ were classified into the CP group, and 157 (62.5\%) were classified into the SC group (Figure 1).

Table 1 shows a comparison of baseline characteristics among the 3 groups. The prevalence of maintenance dialysis was significantly higher in the $\mathrm{CN}$ than other groups. The frequency of statin treatment at discharge was significantly lower in the $\mathrm{CN}$ than other groups. There was a significant difference in serum phosphorus levels among the 3 groups.

Table 2 shows a comparison of lesion and procedural characteristics among the 3 groups. There were significant differences in the frequency of culprit lesions responsible for ACS among the 3 groups. CN was frequently located in the right coronary artery (RCA) and left main trunk. Conversely, CP and SC were frequently located in the left anterior descending artery. The prevalence of severe CAC was significantly higher in the $\mathrm{CN}$ and $\mathrm{CP}$ groups than in the SC group. The use of rotational atherectomy was significantly more common in the $\mathrm{CN}$ group than in the $\mathrm{CP}$ and SC groups. Stent diameter was also significantly greater in the $\mathrm{CN}$ group. Stent length was significantly larger in the $\mathrm{CP}$ than $\mathrm{SC}$ group.

The OCT findings are summarized in Table 3. Maximum calcium arc, maximum calcium thickness, and calcium length were significantly greater in the $\mathrm{CN}$ and $\mathrm{CP}$ groups than in the SC group. Calcium length was significantly greater in the $\mathrm{CN}$ group than in the $\mathrm{CP}$ and $\mathrm{SC}$ groups. There were no significant differences in MLA and MSA among the 3 groups, although the reference lumen area was significantly larger in the $\mathrm{CN}$ group than in the $\mathrm{CP}$ and $\mathrm{SC}$ groups. Total stent length was significantly larger in the $\mathrm{CP}$ than SC group.

The incidence of SED, ISA, and maximum ISA distance were significantly higher in the $\mathrm{CN}$ than SC group.

Clinical follow-up data were obtained for all patients. The median follow-up duration was 728 days (IQR 3171,032 days). The shortest follow-up duration was 1 day because of death or loss to follow-up after discharge. Inhospital death occurred in 3 patients in the SC group and TLR occurred in 1 patient in the SC group and not in the other groups. There were no significant differences in inhospital deaths and TLR between the 3 groups $(\mathrm{P}=0.40$ and $\mathrm{P}=0.74$, respectively).

Figure 3 shows the Kaplan-Meier survival curves for clinical outcomes in the 3 groups. MACE-free survival (log rank, $\mathrm{P}=0.0117$ ), myocardial infarction-free survival (log rank, $\mathrm{P}=0.0103$ ), and TLR-free survival (log rank, $\mathrm{P}=0.0455$ ) were significantly worse in the $\mathrm{CN}$ group. Conversely, survival, cardiac death-free survival, and TVR-free survival were not statistically different among the 3 groups. On the pairwise post hoc tests, the cumulative incidence of MACE was significantly higher in the $\mathrm{CN}$ than $\mathrm{CP}$ $(\mathrm{P}=0.0059)$ and $\mathrm{SC}(\mathrm{P}=0.0132)$ groups, and the cumulative incidence of myocardial infarction was significantly higher in the $\mathrm{CN}$ than $\mathrm{CP}$ group $(\mathrm{P}=0.0043)$. There was no significant difference among each of these pairs in the cumulative incidence of TLR.

To further clarify the prognostic impact of $\mathrm{CN}$ vs. nonCN (CP or SC), we compared patients with and without $\mathrm{CN}$. CN was significantly associated with MACE, myocar- 


\begin{tabular}{|lcccc|}
\hline Table 4. Clinical Outcomes & $\begin{array}{c}\text { Patients with } \mathbf{C N} \\
(\mathbf{n = 2 5 )}\end{array}$ & $\begin{array}{c}\text { Patients without CN } \\
(\mathbf{n = 2 2 6})\end{array}$ & $\begin{array}{c}\text { Hazard ratio } \\
\mathbf{( 9 5 \%} \mathbf{~ C l )}\end{array}$ & P value \\
Primary end point & $7(28.0)$ & $20(8.9)$ & $3.31(1.30-7.48)$ & 0.0146 \\
Secondary end point & & & & \\
All cause death & $2(8.0)$ & $17(7.5)$ & $0.98(0.15-3.41)$ & 0.9756 \\
Cardiac death & $1(4.0)$ & $5(2.2)$ & $1.80(0.09-11.2)$ & 0.6170 \\
MI & $3(12.0)$ & $4(1.8)$ & $6.52(1.28-29.7)$ & 0.0264 \\
Spontaneous MI & $2(8)$ & $3(1.3)$ & $5.96(0.78-36.1)$ & 0.0790 \\
Periprocedural MI & $1(4)$ & $1(0.4)$ & $7.75(0.30-197)$ & 0.1797 \\
TLR & $5(20.0)$ & $13(5.8)$ & $3.41(1.09-9.05)$ & 0.0362 \\
TVR & $6(24.0)$ & $26(11.5)$ & $2.20(0.82-5.01)$ & 0.1096 \\
\hline
\end{tabular}

Unless indicated otherwise, values are presented as $\mathrm{n}(\%)$. Cl, confidence interval; $\mathrm{CN}$, calcified nodule; HR, hazard ratio; MI, myocardial infarction; TLR, target lesion revascularization; TVR, target vessel revascularization.

\begin{tabular}{|c|c|c|c|c|c|c|}
\hline & \multicolumn{2}{|c|}{ Univariate model } & \multicolumn{2}{|c|}{ Multivariate model 1} & \multicolumn{2}{|c|}{ Multivariate model 2} \\
\hline & HR $(95 \% \mathrm{Cl})$ & $P$ value & HR $(95 \% \mathrm{Cl})$ & $P$ value & HR (95\% Cl) & $P$ value \\
\hline $\mathrm{CN}$ & $3.31(1.30-7.48)$ & 0.0146 & $4.23(1.53-10.7)$ & 0.0070 & $4.41(1.63-10.8)$ & 0.0047 \\
\hline Age & $0.99(0.95-1.03)$ & 0.5718 & & & & \\
\hline Male sex & $1.80(0.69-6.14)$ & 0.2460 & & & & \\
\hline Smoking & $0.94(0.41-2.41)$ & 0.8907 & & & & \\
\hline Diabetes & $0.97(0.45-2.08)$ & 0.9464 & & & & \\
\hline Dyslipidemia & $0.61(0.30-1.32)$ & 0.2024 & & & & \\
\hline Hypertension & $0.81(0.33-2.42)$ & 0.6751 & & & & \\
\hline Hemodialysis & $2.84(1.17-6.28)$ & 0.0225 & $1.94(0.71-4.78)$ & 0.1843 & & \\
\hline eGFR & $0.98(0.97-0.99)$ & 0.0031 & & & $0.98(0.97-1.00)$ & 0.0808 \\
\hline LDL-C & $1.01(1.00-1.03)$ & 0.0164 & $1.02(1.00-1.03)$ & 0.0060 & $1.02(1.00-1.03)$ & 0.0097 \\
\hline Statin at discharge & $0.79(0.33-2.16)$ & 0.6145 & & & & \\
\hline ACS & $1.50(0.70-3.22)$ & 0.2932 & & & & \\
\hline Severe calcification & $0.83(0.34-1.84)$ & 0.6615 & & & & \\
\hline Rotational atherectomy & $2.02(0.74-4.74)$ & 0.1570 & & & & \\
\hline Maximum calcium arc & $1.00(0.997-1.01)$ & 0.4201 & & & & \\
\hline Maximum calcium thickness & $1.19(0.29-4.84)$ & 0.8132 & & & & \\
\hline Calcium length & $1.01(0.97-1.05)$ & 0.5356 & & & & \\
\hline Minimum stent area & $1.09(0.87-1.33)$ & 0.4507 & & & & \\
\hline Minimum lumen area & $1.08(0.87-1.32)$ & 0.4796 & & & & \\
\hline Stent underexpansion & $0.96(0.43-2.34)$ & 0.9590 & & & & \\
\hline Stent edge dissection & $1.73(0.75-3.81)$ & 0.1895 & & & & \\
\hline
\end{tabular}

Multivariate Model 1 was adjusted for calcified nodule (CN), maintenance hemodialysis, and serum low-density lipoprotein cholesterol (LDL-C) concentrations. Multivariate Model 2 was adjusted for CN, eGFR, and serum LDL-C concentrations. ACS, acute coronary syndrome; Cl, confidence interval; eGFR, estimated glomerular filtration rate; HR, hazard ratio.

dial infarction, and TLR (Table 4). Table 5 shows the Cox proportional hazards analysis of predictors of MACE. Univariate analysis indicated that $\mathrm{CN}$, maintenance dialysis, estimated glomerular filtration rate, and serum low-density lipoprotein cholesterol (LDL-C) concentrations were significantly associated with MACE. Multivariate analysis including $\mathrm{CN}$, maintenance dialysis, and serum LDL-C concentrations as covariates (Multivariate Model 1) or including $\mathrm{CN}$, estimated glomerular filtration rate, and serum LDL-C concentrations as covariates (Multivariate Model 2) revealed that $\mathrm{CN}$ and serum LDL-C concentrations were independent predictors of MACE.

\section{Discussion}

The major findings of this study are that: (1) in target lesions with moderate to severe CAC, the CN, CP, and SC calcified plaque subtypes were present in $9.9 \%, 26.8 \%$, and $63.3 \%$ of patients, respectively; (2) the prevalence of maintenance hemodialysis was significantly higher in the $\mathrm{CN}$ than $\mathrm{CP}$ and $\mathrm{SC}$ groups, and the prevalence of severe CAC was greatest in the $\mathrm{CN}$ group, followed by the $\mathrm{CP}$ and $\mathrm{SC}$ groups; and (3) MACE occurred more frequently in patients with $\mathrm{CN}$ than in those with $\mathrm{CP}$ or $\mathrm{SC}$, and $\mathrm{CN}$ was an independent predictor of MACE.

Recently, Sugiyama et al ${ }^{\mathbf{1 1}}$ classified calcified plaques responsible for ACS into 3 groups (eruptive $\mathrm{CN}, \mathrm{CP}$, and 
SC) based on OCT findings. These authors reported that the prevalence of $\mathrm{CN}, \mathrm{CP}$, and $\mathrm{SC}$ was $25.5 \%, 7.1 \%$, and $67.4 \%,{ }^{11}$ respectively, whereas in the present study of both ACS and stable coronary artery disease the rates were $9.9 \%, 26.8 \%$, and $63.3 \%$, respectively. Because $\mathrm{CN}$ lesions are characteristic of vulnerable plaques prone to ACS, their prevalence in this study was lower than in the study of Sugiyama et al. However, even after excluding patients with stable coronary artery disease from our study population, the prevalence of $\mathrm{CN}$ remained lower than that reported previously. This discrepancy may be explained by differences in the study populations.

$\mathrm{CN}$ is pathologically defined as the accumulation of small nodular calcifications with disruption of the fibrous cap, and thrombus formation overlying the calcium surface and protruding into the vessel lumen..$^{20,21} \mathrm{CN}$ is recognized as one of the pathogenic mechanisms of ACS. ${ }^{16,20}$ Lee et $\mathrm{al}^{22}$ reported that $\mathrm{CN}$ in PCI culprit lesions was frequently observed in patients on dialysis, in lesions with severe CAC, and in the RCA, especially in the mid or ostial segments. These results are consistent with those of the present study. Although there have been no published reports on pathologically validated $\mathrm{CP}, \mathrm{CP}$ is recognized as a protruding calcified mass similar to $\mathrm{CN}$ on OCT findings.

Newer-generation DES have thinner stent platforms and biocompatible or biodegradable polymers that render these devices less thrombogenic ${ }^{23,24}$ compared with firstgeneration DES. However, target lesions with moderate to severe CAC remain independently associated with adverse outcomes in patients treated with newer-generation DES and modern devices. ${ }^{25}$ Although OCT studies have demonstrated that target lesion calcification limits lumen dilatation by scoring balloon, ${ }^{4}$ stent expansion during PCI, ${ }^{2}$ and neointimal coverage at follow-up, ${ }^{26}$ the association between target lesion CAC and clinical outcome has not been well studied. Shan et $\mathrm{al}^{27}$ reported that there was no significant association between dense calcium volume measured by virtual histology IVUS and TLR or MACE. Conversely, Morofuji et al ${ }^{28}$ reported that $\mathrm{CN}$ by IVUS was significantly associated with MACE, including cardiac death, TLR, and stent thrombosis, in patients with heavily calcified lesions requiring rotational atherectomy. However, IVUS may sometimes confuse $\mathrm{CP}$ with $\mathrm{CN}$ because of its limited resolution. The present study investigated the effect of calcified plaque morphology on clinical outcomes by distinguishing between $\mathrm{CN}$ and $\mathrm{CP}$ using OCT imaging. Sugiyama et al reported that there was no significant difference in in-hospital deaths and TLR among CN, CP, and SC. ${ }^{11}$ The present study also demonstrated no significant difference in in-hospital deaths and TLR among patients with these calcified plaque subtypes, but a significant difference in MACE during long-term follow-up. This was because $\mathrm{CN}$ was significantly associated with MACE that occurred in the chronic phase, driven primarily by a higher rate of TLR and myocardial infarction. To the best of our knowledge, the present study is the first to examine the effects of calcified plaque subtypes on MACE during long-term follow-up after DES implantation.

Stent underexpansion is a well-established predictor of stent failure. ${ }^{18,29}$ Similarly, a previous OCT study reported that MSA was an independent predictor of device-oriented clinical endpoints, including cardiac death, target vesselrelated myocardial infarction, TLR, and stent thrombosis. ${ }^{18}$ In an OCT study targeting patients with ACS, Kobayashi et $\mathrm{al}^{30}$ reported that MSA was smaller in culprit lesions with than without $\mathrm{CN}$, which was associated with higher TLR in CN lesions. Conversely, in the present study, TLR occurred more frequently in patients with $\mathrm{CN}$ despite the greater MSA than in those without CN. In addition, SED, ISA, and stent underexpansion are considered as causes of in-stent restenosis and stent thrombosis. ${ }^{3,31,32} \mathrm{~A}$ recent OCT study reported that $\mathrm{CN}$ was associated with a higher incidence of SED and ISA, ${ }^{12}$ which is consistent with the present study. To clarify the association between post-stent OCT findings and MACE, we compared patients with and without MACE in each calcified plaque subgroup. There was no significant difference in MSA and the incidence of SED, stent underexpansion, and ISA in each calcified plaque subtype (Supplementary Table). Although the mechanism of TLR in lesions with CN has not been clearly identified, some possible causes can be suggested. Previous studies had indicated that $\mathrm{CN}$ was associated with breaks in the calcified sheet and was predominantly located at the mid-RCA, where coronary torsion stress is greater as a result of "hinge motion". ${ }^{20,22}$ This mechanical stress may cause stent fracture, which is related to subsequent stent failure. ${ }^{33}$ Furthermore, a previous human autopsy study suggested a novel mechanism of stent failure caused by $\mathrm{CN}$ from 2 cases where $\mathrm{CN}$ protruded into the lumen after stent implantation. ${ }^{34}$ Therefore, these phenomena after DES implantation, rather than procedural factors such as MSA, stent underexpansion, SED, or ISA, may be related to subsequent stent failure in lesions with $\mathrm{CN}$.

As shown in the present study, calcified lesions with $\mathrm{CN}$ are considered high-risk lesions, even in the era of newergeneration DES. Therefore, alternative strategies to improve the outcome of these high-risk lesions are needed. A recent study reported that TLR rates after drug-coated balloon angioplasty following rotational atherectomy for heavily calcified lesions was comparable to that of newergeneration DES. ${ }^{35}$ Therefore, if appropriate lesion preparation with rotational atherectomy is achieved in heavily calcified lesions with CN, a "stentless" strategy using drugcoated balloons could be an alternative treatment choice. Furthermore, considering the inferior outcomes of target lesions with $\mathrm{CN}$, detection of $\mathrm{CNs}$ in multivessel heavily calcified lesions may encourage the referral of patients for coronary artery bypass grafting rather than performing multiple PCI procedures.

\section{Study Limitations}

Several limitations of this study should be noted. First, this was a single-center retrospective observational study. Second, because individual physicians determined both the treatment strategy and OCT procedure, potential selection bias could not be avoided. At Nara Medical University, OCT-guided PCI is generally avoided in patients with severe renal function not on dialysis and in those with an unstable condition, such as cardiogenic shock or congestive heart failure. Third, OCT assessments after predilatation using a balloon catheter, rotational atherectomy, or thrombectomy were performed in $28.3 \%$ of cases with severe narrowing not amenable to OCT or OFDI catheter passage or removal of blood from the field of view, which may have affected visualization of the calcified plaque. Fourth, OCT may not fully visualize the deep calcium in the setting of superficial plaque attenuation due to its limited depth of penetration. In addition, strong attenuation due to a lipid-rich component within or adjacent to the calcified $\operatorname{area}^{36}$ or a thick calcification may make the outer 
border of the calcification unclear or invisible, resulting in underestimation of the calcium detected by OCT. Fifth, although several studies demonstrated that patients on dialysis had an increased risk of target lesion failure and cardiovascular events after DES implantation, ${ }^{37,38}$ in the present study dialysis was not significantly associated with MACE in the multivariate analysis. It is speculated that this is because dialysis is a strong predictor of $\mathrm{CN}$. Sixth, the sample size of the present present study was relatively small. A large-scale prospective cohort study is needed to investigate the characteristics of $\mathrm{CN}$, which has the greatest effect on future clinical outcomes, and the optimal PCI strategy for calcified lesions with $\mathrm{CN}$.

\section{Conclusions}

Target lesion $\mathrm{CN}$ was associated with more frequent cardiac events in patients who underwent OCT-guided DES implantation for lesions with moderate to severe CAC.

\section{Acknowledgment}

The authors thank the staff of the catheterization laboratory at Nara Medical University for their excellent assistance.

\section{Sources of Funding}

This study did not receive any specific funding.

\section{Disclosures}

Y.S. is a member of Circulation Journal's Editorial Team. The remaining authors have no conflicts of interest to disclose.

\section{IRB Information}

This study was approved by the Ethics Committee of Nara Medical University (Reference no. 1759-2).

\section{Data Availability}

The deidentified participant data will not be shared.

\section{References}

1. Moses JW, Leon MB, Popma JJ, Fitzgerald PJ, Holmes DR, O'Shaughnessy C, et al. Sirolimus-eluting stents versus standard stents in patients with stenosis in a native coronary artery. $N$ Engl J Med 2003; 349: 1315-1323.

2. Kobayash Y, Okura H, Kume T, Yamada R, Kobayashi Y, Fukuhara K, et al. Impact of target lesion coronary calcification on stent expansion. Circ J 2014; 78: 2209-2214.

3. Kuriyama N, Kobayashi Y, Yamaguchi M, Shibata Y. Usefulness of rotational atherectomy in preventing polymer damage of everolimus-eluting stent in calcified coronary artery. JACC Cardiovasc Interv 2011; 4: 588-589.

4. Sugawara Y, Ueda T, Soeda T, Watanabe M, Okura H, Saito Y. Plaque modification of severely calcified coronary lesions by scoring balloon angioplasty using Lacrosse non-slip element: Insights from an optical coherence tomography evaluation. Cardiovasc Interv Ther 2019; 34: 242-248.

5. Matsukawa R, Kozai T, Tokutome M, Nakashima R, Nishimura $\mathrm{R}$, Matsumoto $\mathrm{S}$, et al. Plaque modification using a cutting balloon is more effective for stenting of heavily calcified lesion than other scoring balloons. Cardiovasc Interv Ther 2019; 34: 325-334.

6. Ishibashi Y, Nakatani S, Onuma Y. Definite and probable bioresorbable scaffold thrombosis in stable and ACS patients. EuroIntervention 2015; 11: $\mathrm{e} 1-\mathrm{e} 2$.

7. Généreux P, Madhavan MV, Mintz GS, Maehara A, Palmerini $\mathrm{T}$, Lasalle $\mathrm{L}$, et al. Ischemic outcomes after coronary intervention of calcified vessels in acute coronary syndromes. Pooled analysis from the HORIZONS-AMI (Harmonizing Outcomes With Revascularization and Stents in Acute Myocardial Infarction) and ACUITY (Acute Catheterization and Urgent Intervention Triage Strategy) trials. J Am Coll Cardiol 2014; 63: 1845-1854.

8. Kataiwa H, Tanaka A, Kitabata H, Imanishi T, Akasaka T. Safety and usefulness of non-occlusion image acquisition technique for optical coherence tomography. Circ J 2008; 72: 1536-1537.

9. Kume T, Akasaka T, Kawamoto T, Watanabe N, Toyota E, Sukmawan R, et al. Visualization of neointima formation by optical coherence tomography. Int Heart J 2005; 46: 1133-1136.

10. Kume T, Okura H, Kawamoto T, Yamada R, Miyamoto Y, Hayashida A, et al. Assessment of the coronary calcification by optical coherence tomography. EuroIntervention 2011; 6: 768-772.

11. Sugiyama T, Yamamoto E, Fracassi F, Lee H, Yonetsu T, Kakuta $\mathrm{T}$, et al. Calcified plaques in patients with acute coronary syndromes. JACC Cardiovasc Interv 2019; 12: 531-540.

12. Nakajima A, Araki M, Kurihara O, Minami Y, Soeda T, Yonetsu T, et al. Comparison of post-stent optical coherence tomography findings among three subtypes of calcified culprit plaques in patients with acute coronary syndrome. Catheter Cardiovasc Interv 2021; 97: 634-645.

13. Thygesen K, Alpert JS, Jaffe AS, Simoons ML, Chaitman BR, White HD, et al. Third universal definition of myocardial infarction. Circulation 2012; 126: 2020-2035.

14. Watanabe M, Uemura S, Sugawara Y, Ueda T, Soeda T, Takeda $\mathrm{Y}$, et al. Side branch complication after a single-stent crossover technique: Prediction with frequency domain optical coherence tomography. Coron Artery Dis 2014; 25: 321-329.

15. Fujii K, Kubo T, Otake H, Nakazawa G, Sonoda S, Hibi K, et al. Expert consensus statement for quantitative measurement and morphological assessment of optical coherence tomography. Cardiovasc Interv Ther 2020; 35: 13-18.

16. Sugiura J, Watanabe M, Toyokawa N, Kamon D, Isojima T, Ueda T, et al. Progression of a calcified nodule causing acute myocardial infarction in a patient on hemodialysis: Serial optical coherence tomography. Circ J 2019; 83: 490.

17. Wang X, Matsumura M, Mintz GS, Lee T, Zhang W, Cao Y, et al. In vivo calcium detection by comparing optical coherence tomography, intravascular ultrasound, and angiography. JACC Cardiovasc Imaging 2017; 10: 869-879.

18. Soeda T, Uemura S, Park SJ, Jang Y, Lee S, Cho JM, et al. Incidence and clinical significance of poststent optical coherence tomography findings: One-year follow-up study from a multicenter registry. Circulation 2015; 132: 1020-1029.

19. Moussa ID, Klein LW, Shah B, Mehran R, Mack MJ, Brilakis ES, et al. Consideration of a new definition of clinically relevant myocardial infarction after coronary revascularization an expert consensus document from the Society for Cardiovascular Angiography and Interventions (SCAI). J Am Coll Cardiol 2013; 62: $1563-1570$.

20. Virmani R, Kolodgie FD, Burke AP, Farb A, Schwartz SM. Lessons from sudden coronary death: A comprehensive morphological classification scheme for atherosclerotic lesions. Arterioscler Thromb Vasc Biol 2000; 20: 1262-1275.

21. Alfonso F, Joner M. Untangling the diagnosis and clinical implications of calcified coronary nodules. JACC Cardiovasc Imaging 2017; 10: 892-896.

22. Lee T, Mintz GS, Matsumura M, Zhang W, Cao Y, Usui E, et al. Prevalence, predictors, and clinical presentation of a calcified nodule as assessed by optical coherence tomography. JACC Cardiovasc Imaging 2017; 10: 883-891.

23. Kolandaivelu K, Swaminathan R, Gibson WJ, Kolachalama VB, Nguyen-Ehrenreich KL, Giddings VL, et al. Stent thrombogenicity early in high risk interventional settings is driven by stent design and deployment, and protected by polymer-drug coatings. Circulation 2011; 123: $1400-1409$.

24. Joner M, Finn AV, Farb A, Mont EK, Kolodgie FD, Ladich E, et al. Pathology of drug-eluting stents in humans: Delayed healing and late thrombotic risk. J Am Coll Cardiol 2006; 48: 193-202.

25. Copeland-Halperin RS, Baber U, Aquino M, Rajamanickam A, Roy S, Hasan C, et al. Prevalence, correlates, and impact of coronary calcification on adverse events following PCI with newer-generation DES: Findings from a large multiethnic registry. Catheter Cardiovasc Interv 2018; 91: 859-866.

26. Ueda T, Uemura S, Watanabe M, Dote Y, Goryo Y, Sugawara $\mathrm{Y}$, et al. Thin-cap fibroatheroma and large calcification at the proximal stent edge correlate with a high proportion of uncovered stent struts in the chronic phase. Coron Artery Dis 2016; 27: 376-384.

27. Shan P, Mintz GS, Witzenbichler B, Metzger DC, Rinaldi MJ, Duffy PL, et al. Does calcium burden impact culprit lesion morphology and clinical results?: An ADAPT-DES IVUS substudy. Int J Cardiol 2017; 248: 97-102.

28. Morofuji T, Kuramitsu S, Shinozaki T, Jinnouchi H, Sonoda S, Domei T, et al. Clinical impact of calcified nodule in patients 
with heavily calcified lesions requiring rotational atherectomy. Catheter Cardiovasc Interv 2021; 97: 10-19.

29. Fujii K, Carlier SG, Mintz GS, Yang YM, Moussa I, Weisz G, et al. Stent underexpansion and residual reference segment stenosis are related to stent thrombosis after sirolimus-eluting stent implantation: An intravascular ultrasound study. J Am Coll Cardiol 2005; 45: 995-998.

30. Kobayashi N, Takano M, Tsurumi M, Shibata Y, Nishigoori S, Uchiyama S, et al. Features and outcomes of patients with calcified nodules at culprit lesions of acute coronary syndrome: An optical coherence tomography study. Cardiology 2018; 139: 90-100.

31. Mosseri M, Satler LF, Pichard AD, Waksman R. Impact of vessel calcification on outcomes after coronary stenting. Cardiovasc Revasc Med 2005; 6: 147-153.

32. Kume T, Okura H, Miyamoto Y, Yamada R, Saito K, Tamada $\mathrm{T}$, et al. Natural history of stent edge dissection, tissue protrusion and incomplete stent apposition detectable only on optical coherence tomography after stent implantation: Preliminary observation. Circ J 2012; 76: 698-703.

33. Kuramitsu S, Iwabuchi M, Haraguchi T, Domei T, Nagae A, Hyodo M, et al. Incidence and clinical impact of stent fracture after everolimus-eluting stent implantation. Circ Cardiovasc Interv 2012; 5: 663-671.
34. Mori H, Finn AV, Atkinson JB, Lutter C, Narula J, Virmani R. Calcified nodule: An early and late cause of in-stent failure. JACC Cardiovasc Interv 2016; 9: e125-e126.

35. Ueno K, Morita N, Kojima Y, Takahashi H, Kawasaki M, Ito $\mathrm{R}$, et al. Safety and long-term efficacy of drug-coated balloon angioplasty following rotational atherectomy for severely calcified coronary lesions compared with new generation drug-eluting stents. J Interv Cardiol 2019; 2019: 9094178.

36. Ijichi T, Nakazawa $G$, Torii S, Nakano M, Yoshikawa A, Morino Y, et al. Evaluation of coronary arterial calcification: Ex-vivo assessment by optical frequency domain imaging. Atherosclerosis 2015; 243: 242-247.

37. Kume T, Uemura S. Late failure of first-generation drug-eluting stents in hemodialysis patients. Circ J 2015; 79: 2103-2105.

38. Lee JM, Kang J, Lee E, Hwang D, Rhee TM, Park J, et al. Chronic kidney disease in the second-generation drug-eluting stent era: Pooled analysis of the Korean Multicenter Drug-Eluting Stent Registry. JACC Cardiovasc Interv 2016; 9: 2097-2109.

\section{Supplementary Files}

Please find supplementary file(s);

http://dx.doi.org/10.1253/circj.CJ-20-1233 